\title{
USE OF CODECS IN VIDEO UPLOADS
}

\author{
Andrej Učakar (D), Patricija Selič (D), Raša Urbas (D) \\ University of Ljubljana, Faculty of Natural Sciences and Engineering, Department of Textiles, \\ Graphis Arts and Design, Chair of Information and Graphic Arts Technology, Ljubljana, Slovenia
}

\begin{abstract}
In recent decades, linear communication has largely been replaced by visual depictions that contain not only moving images but also sound, text and other (visible or invisible) data. It is therefore not surprising that video has become so popular, especially among young people. So-called multimedia communication is transmitted via a variety of media. Since there are many ways and means of transmission, we wanted to investigate how the type of encoding that allows efficient file reduction affects the characteristics of the video format. For this purpose, we encoded selected videos using the three most common encoding mechanisms H.264/AVC, MPEG-2 and H.265/HEVC at high and medium bitrate. The newly created video files were then uploaded to three popular platforms - Instagram, YouTube and Gmail. Changes in file size, colour gamut, frame and bitrate, resolution and other visually recognizable details are presented in the paper.
\end{abstract}

Key words: codec, compression, encoding, file format, upload, video

\section{INTRODUCTION}

Video contents have been a part of our daily lives for almost half a century. Since their beginnings in the early 1980s' they have developed not only in terms of content but also in terms of quality. The integration of moving images, text, sound and sometimes other effects has determined the ever-increasing size and consequently the complexity of written files. Improved recording quality, higher frame rate (fps), resolution (ppi) and quality of rendering demand a sophisticated development of different codecs and file types, which enable higher video quality, smaller file size and improved rendering mode.

In order to either share or post videos for viewing on different screens and devices, in small or large dimensions, video content must be recorded in a variety of different formats. In an effort to make video content as realistic as possible, the use of compression and interpolation methods in formatting (without noticeable data loss) is essential (Rippel et al, 2018; Le Tanou and Blestel, 2019; Laude et al, 2017). Optimal results are controlled by the use of codecs (short for coder/decoder), which are stored in more complex containers that properly compress the video content and its components (audio/metadata) in each file. Codecs encode audio and/or video into a stream of bytes. Choosing the right codec is based on several variables - digital storage space, frame and bit rate, encoding mode, resolution, colour gamut and others. Today, different codecs are used, each with its own advantages and disadvantages. The selection of the appropriate codec therefore depends on the type of transmission, either via an external hard drive, by e-mail or by uploading to various websites and platforms. The upload requirements depend on the type of codecs supported by either the devices or platforms. These restrictions condition the use of certain codecs. Until 2013, the market for standardized video codecs was dominated by ISO (International Organization for Standardization), IEC (International Electrotechnical Commission) and ITU-T (Telecommunication Standardization Sector) standardization groups, with most representable codecs such as MPEG-1, MPEG2/H.262, H.263, MPEG-4 Visual and Advanced Video Coding (i.e. AVC, also known as MPEG-4 Part 10 or H.264) (Laude et al, 2017; Zhang and Mao, 2018; The Moving Picture Experts Group, n.d.). These standards have most of the basic functions in common, but differ in detail. In general H.264 provides better compression efficiency and has greater flexibility in compressing, transmitting and storing video than MPEG-1 and MPEG-2. Advances in video coding have resulted in high efficiency video coding (i.e. HEVC), which has been standardized by ISO/IEC as MPEG-H Part 2 and by ITU-T as H.265 (Richardson, 2017).

In this scientific paper we present the differences in the characteristics of selected videos compressed with MPEG-2, H.264/AVC and H.265/HEVC codecs. The aim of the study was to determine how the codec type affects the properties of video recording (i.e. visual presentation (occurrence of unwanted image artifacts), file size, frame and bit rate, resolution, colour gamut and other properties) when displayed on different platforms - Instagram, YouTube, and Gmail. 


\section{VIDEO PREPARATION AND PROCESSING}

Video preparation and processing. For video recording we used the compact system camera Sony Alpha A5000 (Sony, Japan) with 20 megapixel APS-C CMOS (e.g. complementary metal-oxide-semiconductor) image sensor. The device allows maximum video HD resolution of $1920 \times 1080$ pixels, with 50i, 25p or 24p $\mathrm{fps}$. Videos can be saved in two types of video containers - MP4 and AVCHD, both using the same codec H.264/AVC.

For the acquisition of the individual video image frames, the comparison of the histograms and the conversion into different codecs, the Adobe Photoshop and Adobe Media Encoder software were used. The VLC media player was used for verification and comparison of the stored video versions.

Video recording. For the purposes of this study, videos were recorded at maximum camera resolution, aspect ratio $16: 9,25 \mathrm{fps}$ in progressive scan mode, at an average bitrate of $17 \mathrm{Mbps}$, and maximum colour gamut. The video was recorded during the day when there is enough natural light. The decision was to record a video of blooming, colourful flowers, which allowed a wide range of colour shades to be captured. Several different videos were recorded, both from a distance and close up, with the focus object resting or moving in the wind. In addition, the recording time also varied - from a few seconds to a minute - with the aim of selecting the most suitable video for comparison and analysis.

Variations of compared videos. Among the recorded videos we chose the one with the most details and the maximum colour gamut (mainly because of its numerous shades of green). The video had 00011.mts (MPEG-2 Transport Stream; M2TS) and took up 27.1 MB of memory. It was 13 seconds long, recorded at maximum resolution, at the final bitrate of $6.3 \mathrm{Mbps}$ and encoded using the H.264/AVC codec. Three file formats, i.e. codecs, were used with the VBR (variable bitrate) encoding method and different presets that allowed different video storage characteristics i.e. bitrate settings (Table 1).

Table 1: Video conversion properties and corresponding upload platforms

\begin{tabular}{|c|c|c|c|c|c|}
\hline Codec & \multicolumn{2}{|c|}{ H.264/AVC } & \multicolumn{2}{|c|}{ MPEG-2 } & H.265/HEVC \\
\hline Bitrate (Mbps) & $\begin{array}{c}\text { 10.0-12.0 } \\
\text { (high) }\end{array}$ & $\begin{array}{c}3.0-6.0 \\
\text { (medium) }\end{array}$ & $\begin{array}{c}4.0-18.5 \\
\text { (high) }\end{array}$ & $\begin{array}{c}2.5-6.0 \\
\text { (medium) }\end{array}$ & $\begin{array}{c}\text { 16.0-20.0 } \\
\text { (high) }\end{array}$ \\
\hline Upload platform & $\begin{array}{l}\text { O } \\
\text { Instagram } \\
\text { YouTube } \\
\text { M }\end{array}$ & $\begin{array}{l}\text { O } \\
\text { Instagram } \\
\text { YouTube } \\
\text { Gmail }\end{array}$ & $\begin{array}{l}\text { YouTube } \\
\text { M }\end{array}$ & $\begin{array}{l}\text { YouTube } \\
\text { Gmail }\end{array}$ & $\begin{array}{l}\text { O } \\
\text { Instagram } \\
\text { YouTube } \\
\end{array}$ \\
\hline
\end{tabular}

Video upload. For research purposes, all five videos were uploaded to Instagram, YouTube and Gmail platforms. Due to the fact that all three platforms have individual requirements and limitations, the procedures for uploading videos differed (Table 1).

To upload to Instagram, we first had to create a user profile. Later we transferred the selected videos to the smartphone (Huawei p20 lite) using a USB cable (drag and drop from one file folder to another). Even though the mobile application for video posting does not support screen rotation and the $16: 9$ aspect ratio (it only allows $4: 5$ ), the videos could be uploaded unchanged. Unfortunately, the original video could not be uploaded to the smartphone because the device does not have the codecs to read .mts file formats. Instagram only supports H.264/AVC and H.265/HEVC codecs and not MPEG-2, so the later video conversions were not performed. Size limits are set at $650 \mathrm{MB}$ for videos that last 10 minutes or less and 3.6 GB for videos up to 60 minutes in length (valid for 2020; constraints often change with the development of ICT).

To upload to YouTube, videos were simply uploaded to the platform and published privately. Since they were not published publicly, the videos were only visible in Beta Studio of our YouTube channel. All five videos were uploaded without any problems and later transferred back to the computer. When uploading to YouTube, only the size is limited (128 GB, HD video).

Gmail has a certain size limit for sending files, which cannot exceed $25 \mathrm{MB}$ in a single message. Therefore, only videos encoded using the H.264/AVC and MPEG-2 medium bitrate codecs met this criterion. Both videos were dragged into the application and sent as a new email when uploaded. After receiving the mail, the videos were saved on the computer. Gmail also offers the possibility of sending files larger than $25 \mathrm{MB}$, 
but in this case the files must be uploaded to Google Drive (cloud storage service, limited in size according to the individual user). We wanted to specify if the videos are re-encoded when uploaded to Google Drive. The remaining three videos (encoded with the H.264/AVC and MPEG-2 high bitrate, and H.265/HEVC) were sent with Gmail in the same way as before (although before pre-uploaded to Google Drive), although their size was now larger (26, 28 and $30 \mathrm{MB})$. The received files were then stored on the computer.

Video comparison. The comparison of the selected five videos was based on the resulting changes in video conversions (original and re-encoded), which were reflected in alterations of video file sizes, different bit rates and smaller colour gamut, as well as the occurrence of unwanted artifacts due to compression and interpolation. Since colour changes in the videos (due to movement) could not be observed accurately, we performed this comparison on a defined static part of the videos - video image frame (marked with red square in Figure 1), at high magnification (1300\%).

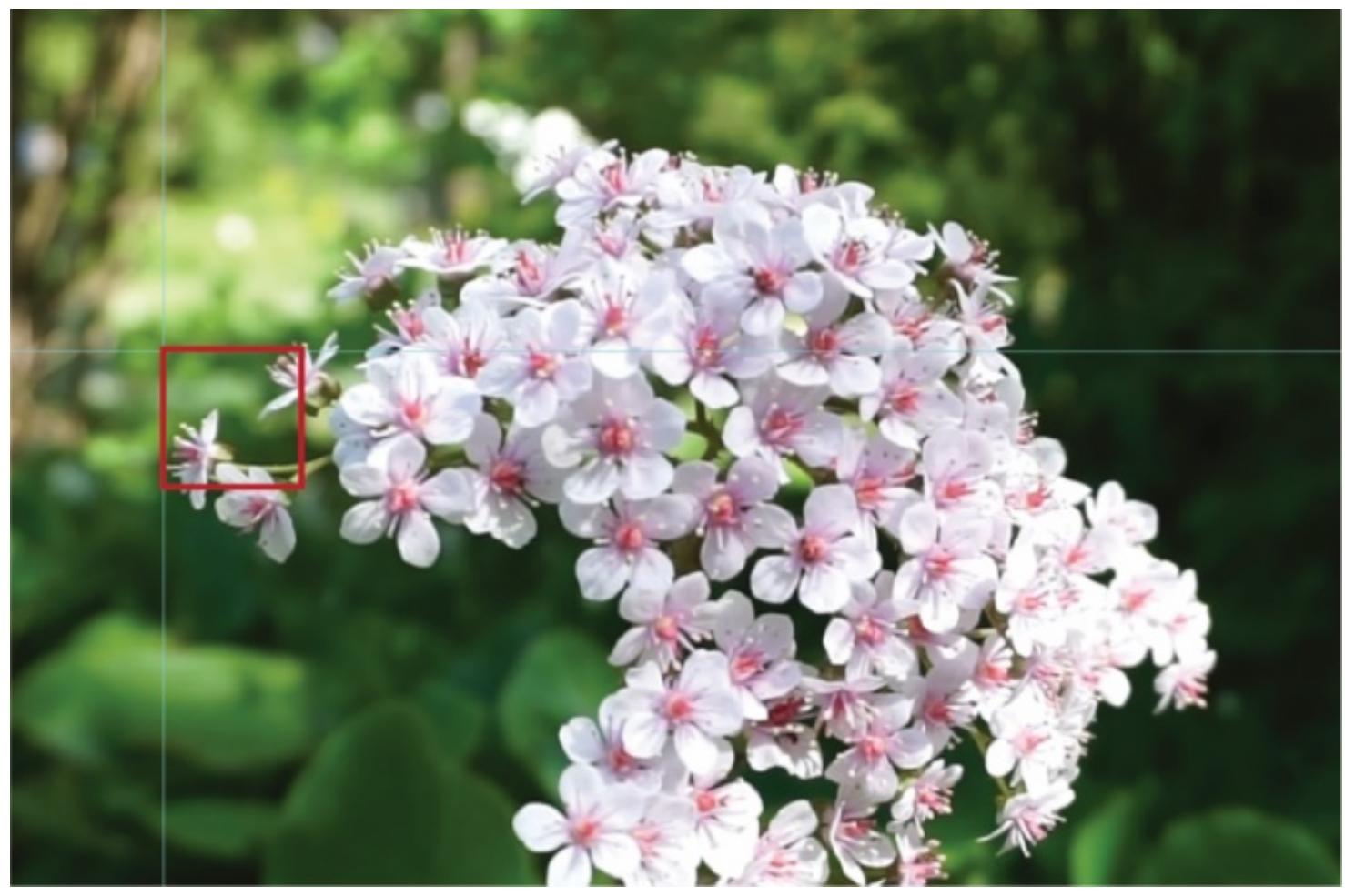

Figure 1: Example of selected video image frame

The colour differences were determined by comparing the histograms of the individual enlarged image frames. For this purpose, Adobe Photoshop was used, where each converted image frame was copied as a separate layer into a new document. By changing the visibility of each layer, we observed differences in the histograms and channel views.

\section{RESULTS AND DISCUSSION}

\subsection{Results of video format data changes}

As expected, the use of different encoding methods (i.e. codecs) had a significant impact on video file properties (Table 2). For high bitrate encoding methods, the file size of H.265/HEVC (10.83\%) and H.264/AVC (6.59\%) codecs increased, while the file size of MPEG-2 codec decreased slightly (3.92 \%). An opposite effect was observed for bitrate data, where all three high bitrate encoding methods increased in size - in the case of MPEG-2 the increase in bitrate was quite significant (11.65\%) and slightly less for H.265/HEVC (8.06 \%), while for H.264/AVC the increase was almost equal (1.55\%).

The use of medium bitrate encoding methods led to more drastic changes in video file properties. Both file size and bitrate decreased significantly for about $69 \%$ (using the medium bitrate encoding method H.264/AVC, file size decreased for $68.17 \%$ and bitrate for $69.19 \%$ ), while using the medium MPEG-2 encoding method resulted in a slightly smaller change of $62 \%$ (file size for $62.76 \%$ and bitrate for $61.92 \%)$. 
The results are rather illogical with regard to selected coding methods. In fact, we expected that video encoded with the H.265/HEVC codec (as the most efficient method) would produce the smallest files, followed by H.264/AVC and MPEG-2 at high and medium bitrate, respectively. Due to the fact that all video conversions were performed in Adobe Media Encoder, with used presets, we are not quite sure which compression mechanisms and interpolation algorithms were used. We assume that this could be the reason for the results. From the above we could therefore conclude that it would be necessary to further analyse and determine the causes of the situation.

As will be shown in the continuation, the differences found led to visible changes in colour transformations (reduction in the number of colour shades and coarser colour transitions) and the occurrence of unwanted artifacts in the form of aliasing, blurring and edge halo.

Interestingly encoding (regardless of bitrate speed) increased the length of the videos by one second, adding a black image frame at the end of videos. The reason for this was not found.

Table 2: Changes in video file properties after using different encoding methods

\begin{tabular}{|c|c|c|c|c|c|}
\hline Codec & \multicolumn{2}{|c|}{ H.264/AVC } & \multicolumn{2}{|c|}{ MPEG-2 } & \multirow{2}{*}{$\begin{array}{c}\text { H.265/HEVC } \\
\begin{array}{c}16.000-20.000 \\
\text { (high) }\end{array}\end{array}$} \\
\hline Bitrate (bps) & $\begin{array}{c}\text { 10.000-12.000 } \\
\text { (high) }\end{array}$ & $\begin{array}{c}3.000-6.000 \\
\text { (medium) }\end{array}$ & $\begin{array}{c}4.000-18.500 \\
\text { (high) }\end{array}$ & $\begin{array}{c}2.500-6.000 \\
\text { (medium) }\end{array}$ & \\
\hline Original container type & \multicolumn{5}{|c|}{ mts } \\
\hline Encoded container & \multicolumn{2}{|c|}{ MP4 } & \multicolumn{2}{|c|}{ MPEG } & MP4 \\
\hline Original video length (s) & \multicolumn{5}{|c|}{13} \\
\hline Encoded video length (s) & 14 & 14 & 14 & 14 & 14 \\
\hline Original file size $(\mathrm{kB})$ & \multicolumn{5}{|c|}{27.100} \\
\hline Encoded file size (kB) & 28.390 & 8.625 & 26.037 & 10.091 & 30.393 \\
\hline Original bitrate $(\mathrm{kb} / \mathrm{s})$ & \multicolumn{5}{|c|}{16.344} \\
\hline Encoded bitrate $(\mathrm{kb} / \mathrm{s})$ & 16.601 & 5.036 & 18.500 & 6.224 & 17.776 \\
\hline
\end{tabular}

\subsection{Results of video format visual changes}

The results of the encoded videos differed from the original video in colour gamut and image quality. These changes were noticeable not only in the histograms, but also in the appearance of unwanted artifacts (mainly jagged details in enlarged video images, blur and reduced saturation (Figure 2 and Figure 3).

The colour differences were not the same for all coding methods used. As expected, these changes were more pronounced in highly compressed video files with smaller file sizes. Good visual results were achieved with the H.265/HEVC codec, which had bright and saturated colours, smooth gradients, and few unwanted artifacts. The jagged edges were less intense than in the original video, especially in the greens (Figure 3). A slightly better quality was achieved with high bitrate H.264/AVC encoding, although histograms show similar colour changes as H.265/HEVC encoded video. The worst results were obtained with videos encoded using medium and high bitrate MPEG-2 codecs, where colour transformations were strongest. The compression mechanisms greatly reduced the number of colour shades (especially in the greens) and the interpolation algorithms caused numerous artefacts that resulted in aliasing and high blur (Figure 3). These artefacts occurred not only in selected video image frames, but also in the video.

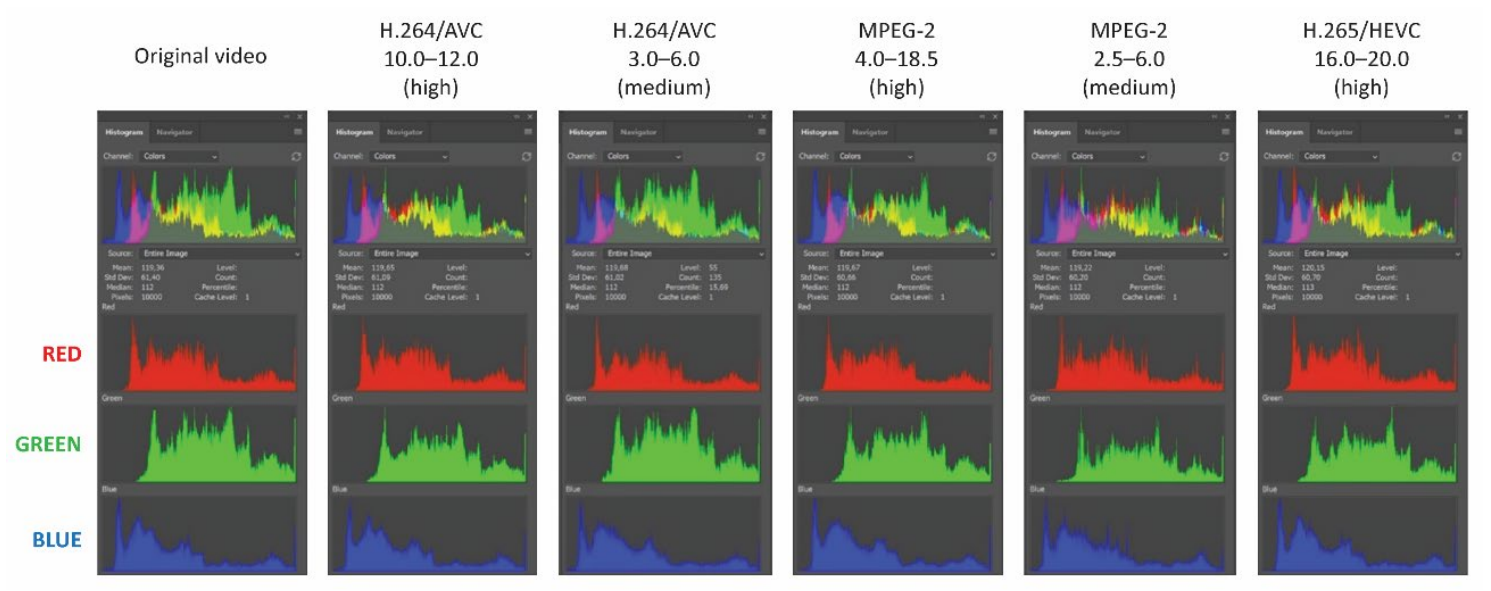

Figure 2: Histograms of original and encoded videos with different high and medium codecs 


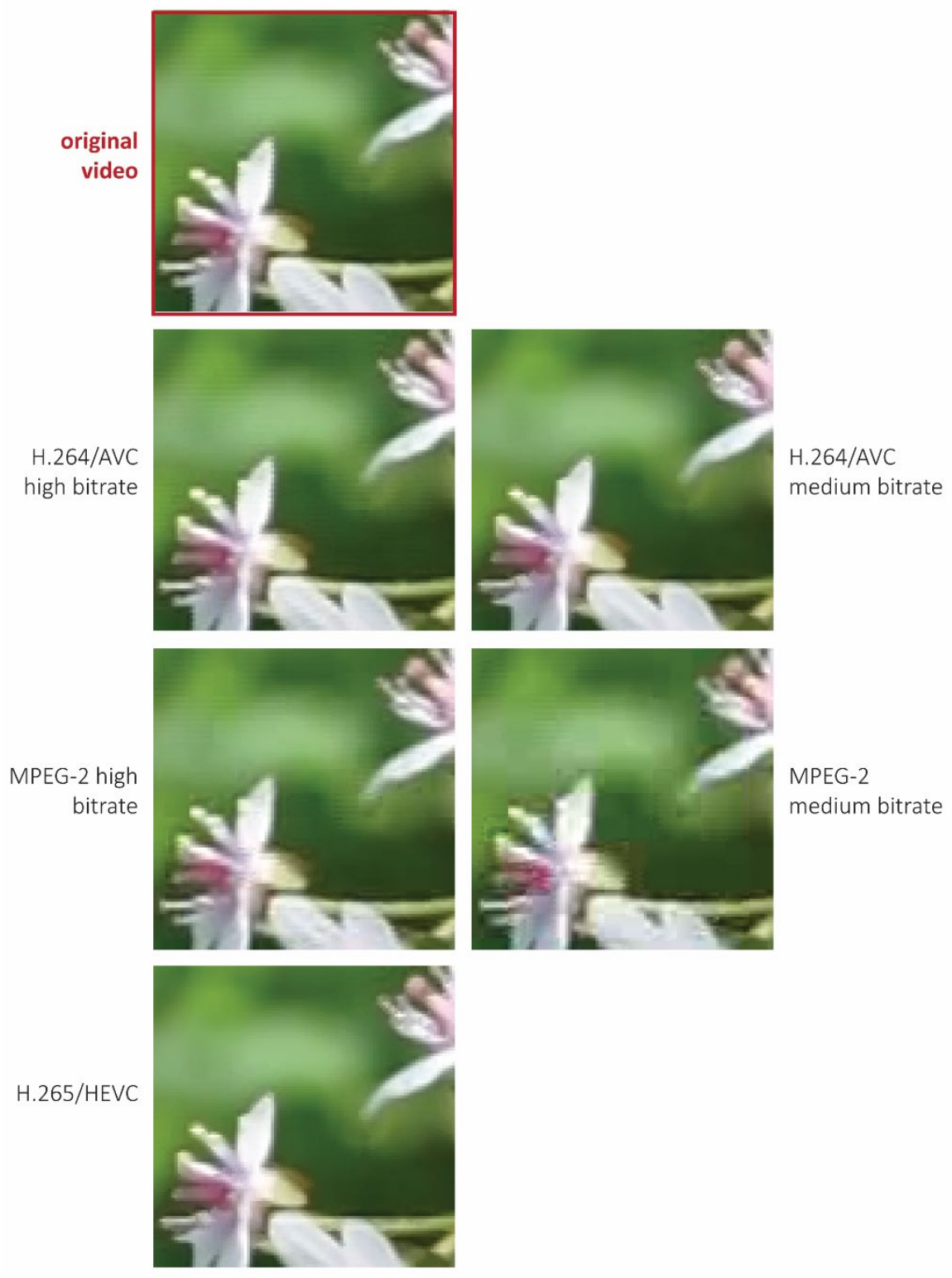

Figure 3: Enlarged details (1300\%) of encoded selected video image frames

\subsection{Results of platforms video uploads}

When uploading content to platforms, each platform uses its own algorithms and settings that determine the properties of the uploaded files (Table 3).

As mentioned above, Instagram uses predefined encoding methods (it only supports the H.264/AVC and H.265/HEVC codecs and changes them to MP4), so uploading with MPEG-2 is not possible) and reduces the resolution while keeping the bitrate unchanged. As the resolution decreases during upload, the file size (at the same bitrate) is reduced accordingly. Since Instagram is primarily a mobile application, we observed the changes in all the videos first on the smartphone and secondly on the computer. The changes in video file sizes were high, most noticeable for the most efficient H.265/HEVC codec (91.05\%), and slightly less for H.264/AVC with high (88.86 \%) and medium bitrate (67.77\%), while the resolution decreased by 62.5 $\%$. The visual changes observed on a smartphone were small but noticeable. As expected, the video encoded with H.264/AVC at medium bitrate had the most flaws. The edges were more jagged due to the lower bitrate and the colour transitions were not as smooth. The other two videos (high bitrate H.264/AVC and H.265/HEVC) looked good, with smooth colour transitions, no aliasing and jagged edges. H.264/AVC encoded video had slightly more saturated colours. The visual changes mentioned were even more noticeable when viewed on a computer due to the larger screen size and aspect ratio and the use of codecs and algorithms adapted for mobile applications. 
Based on the results, we would like to emphasize that when uploading videos to Instagram, it is most advisable to adjust the properties of the files to be uploaded as closely as possible to the characteristics of that platform, and also invert the aspect ratio $(1080 \times 1920 \mathrm{px})$.

We found that all videos uploaded to YouTube were encoded using the H.264/AVC codec, which has been changed to the MP4 container. This platform is one of the few that allows arbitrary selection of viewing resolution, even in full resolution. Despite the above, the resolution in encoded videos decreased (by 33.33 $\%)$, although not as much as in the encoding for Instagram. After uploading all encoded videos, the file size was reduced to about $4.000 \mathrm{~KB}$ at an average bitrate of $128 \mathrm{~kb} / \mathrm{s}$. When we upload the video to YouTube, the compression mechanisms change it regardless of the format and resolution used. It is therefore recommended that we are aware of these changes and keep them to a minimum. Visible changes were the smallest in the high bitrate H.264/AVC codec because the platform uses the same codec for encoding. It is recommended that you choose a slightly larger file size and bitrate when uploding videos so that the platform is able to handle more data that is reduced and changed due to the encoding process. It's also important that we do not encode videos with too much data, as this would require a more extensive encoding, while the quality of the video will not be better. On the other hand, if the amount of data is insufficient, the video quality cannot be restored. When uploading to YouTube, we must also consider the bandwidth of the Internet - if it is too weak, the quality of a video will be in lower resolution.

The study has shown that when uploading to YouTube, we should use an H.264/AVC codec in a high bitrate MP4 container, with the resolution remaining the same as in the original video (in our case: $1920 \times 1080 \mathrm{px})$.

There are two ways to send videos via Gmail. The first way is to share videos as an attachment, with the video file size limited to $25 \mathrm{MB}$, and the second way is to upload the video file to a cloud Google Drive storage first. Sending videos in Gmail requires high compression, which results in high data loss. As the file size decreases, the video quality deteriorates dramatically. We have found that in the case of our encoded videos only two are suitable - H.264/AVC and MPEG-2 at medium bitrate, with encoding resulting in file sizes smaller than $25 \mathrm{MB}$. When comparing the two video qualities, we found that H.264/AVC can provide better visual results. For video encoded with MPEG-2, we noticed jagged edges, blur, and uneven and unnatural colour gradients. Due to the poor quality, this method is therefore only suitable for sending video previews. On the other hand, if we want to share videos in high quality, it is necessary to upload the video files to Google Drive, where the content will remain unchanged, i.e. not re-encoded, while only a link to the video file will be sent by mail. There are (theoretically) no limitations here.

Table 3: Pre- and post-upload properties of video files encoded with different codecs

\begin{tabular}{|c|c|c|c|c|c|c|c|}
\hline \multirow[b]{2}{*}{ Platform } & \multicolumn{2}{|r|}{ Codec } & \multicolumn{2}{|c|}{ H.264/AVC } & \multicolumn{2}{|c|}{ MPEG-2 } & \multirow{2}{*}{$\begin{array}{c}\text { H.265/HEVC } \\
16.000- \\
20.000 \\
\text { (high) } \\
\end{array}$} \\
\hline & $\begin{array}{l}\text { Video } \\
\text { properties }\end{array}$ & $\begin{array}{l}\text { Bitrate } \\
\text { (Mbps) }\end{array}$ & $\begin{array}{c}10.000- \\
12.000 \\
\text { (high) }\end{array}$ & $\begin{array}{c}3.000- \\
6.000 \\
\text { (medium) }\end{array}$ & $\begin{array}{l}4.000- \\
18.500 \\
\text { (high) }\end{array}$ & $\begin{array}{c}2.500- \\
6.000 \\
\text { (medium) }\end{array}$ & \\
\hline \multirow{6}{*}{ Instagram } & \multirow{2}{*}{ File size (kB) } & pre-upload & 28.390 & 8.625 & \multirow{4}{*}{$\begin{array}{c}\text { not } \\
\text { possible }\end{array}$} & \multirow{4}{*}{$\begin{array}{c}\text { not } \\
\text { possible }\end{array}$} & 30.393 \\
\hline & & post-upload & 3.164 & 2.780 & & & 2.719 \\
\hline & \multirow{2}{*}{\begin{tabular}{|l} 
Average \\
bitrate $(\mathrm{kb} / \mathrm{s})$
\end{tabular}} & pre-upload & \multirow{2}{*}{319} & \multirow{2}{*}{126} & & & \multirow{2}{*}{190} \\
\hline & & post-upload & & & & & \\
\hline & \multirow{2}{*}{$\begin{array}{l}\text { Resolution } \\
(\mathrm{px})\end{array}$} & pre-upload & \multicolumn{5}{|c|}{$1920 \times 1080$} \\
\hline & & post-upload & \multicolumn{5}{|c|}{$720 \times 406$} \\
\hline \multirow{6}{*}{ YouTube } & \multirow{2}{*}{ File size (kB) } & pre-upload & 28.397 & 8.625 & 26.037 & 10.091 & 30.393 \\
\hline & & post-upload & 4.010 & 4.007 & 4.023 & 4.015 & 3.820 \\
\hline & \multirow{2}{*}{$\begin{array}{l}\text { Average } \\
\text { bitrate }(\mathrm{kb} / \mathrm{s})\end{array}$} & pre-upload & 319 & 126 & 284 & 224 & 190 \\
\hline & & post-upload & \multicolumn{5}{|c|}{128} \\
\hline & \multirow{2}{*}{$\begin{array}{l}\text { Resolution } \\
(\mathrm{px})\end{array}$} & pre-upload & \multicolumn{5}{|c|}{$1920 \times 1080$} \\
\hline & & post-upload & \multicolumn{5}{|c|}{$1280 \times 720$} \\
\hline \multirow{3}{*}{ M Gmail } & \multirow{2}{*}{ File size (kB) } & pre-upload & \multirow{3}{*}{$\begin{array}{c}\text { not } \\
\text { possible }\end{array}$} & 8.625 & \multirow{3}{*}{$\begin{array}{c}\text { not } \\
\text { possible }\end{array}$} & 10.091 & \multirow{3}{*}{$\begin{array}{c}\text { not } \\
\text { possible }\end{array}$} \\
\hline & & post-upload & & & & & \\
\hline & $\begin{array}{l}\text { Average } \\
\text { bitrate }(\mathrm{kb} / \mathrm{s})\end{array}$ & $\begin{array}{l}\text { pre-upload } \\
\text { post-upload }\end{array}$ & & 126 & & 224 & \\
\hline \multirow{4}{*}{ M } & \multirow{2}{*}{ File size (kB) } & pre-upload & \multirow[b]{2}{*}{28.397} & \multirow{2}{*}{8.625} & & & \\
\hline & & post-upload & & & 26.037 & 10.091 & 30.393 \\
\hline & Average & pre-upload & 319 & 126 & 284 & 224 & 190 \\
\hline & bitrate $(\mathrm{kb} / \mathrm{s})$ & post-upload & 319 & 126 & 284 & 224 & 190 \\
\hline
\end{tabular}




\section{CONCLUSIONS}

The results showed that the most suitable video codec for video display is H.264/AVC. It allows bitrate and resolution changes and is also generally the most commonly used video encoding method since 2019. As it is a lossy compression method, its transformations are perceived, although they are usually imperceptible to the human eye, especially when displaying video (moving images). Similar results have been achieved with the H.265/HEVC codec, which is somewhat larger in file size. Saving in MPEG-2 codecs (regardless of bitrate) resulted in noticeable unnatural colour gradients and blurred images (aliasing), larger changes in file size and bitrate.

The most suitable video format for uploading to YouTube is the H.264/AVC codec in MP4 format with high bitrate, but when uploading the video file to Instagram we must also take into account the resolution and aspect ratio, which are limited. The least limited is uploading via Gmail, as we can upload the file to the cloud and only send access to it. However, if we only want to send via Gmail, we have to use a lower bitrate, which reduces the file size to a maximum of $25 \mathrm{MB}$.

In this research, the Adobe Media Encoder with coding parameters set as default presets was used. Since the comparison of the video encoding methods led to quite unexpected results, a more detailed analysis is planned with the help of open source programs, which allow manual setting of certain parameters and their control. We hope that their use will help us to answer the question why the file sizes are so large when using the H.265/HEVC codec.

\section{REFERENCES}

[1] Laude, T., Adhisantoso, Y. G., Voges, J., Munderloh, M., Ostermann, J.: "A Comprehensive Video Codec Comparison", SIP 8 (30), 1-16, 2017. doi: 10.1017/ATSIP.2019.23

[2] Le Tanou, J., Blestel, M.: "Analysis of Emerging Video Codecs: Coding Tools, Compression Efficiency and Complexity", Society of Motion Picture \& Television Engineers Technical Conference SMPTE 2018, (SMPTE 2018: Los Angeles CA, USA, 2019).

[3] Richardson, I. E.: "Video compression codecs: a survival guide", IASA - International Association of Sound and Audiovisual Archives Journal 47, 1-8,2017. doi: 10.35320/ij.v0i47.51

[4] Rippel, O., Nair, S., Lew, C., Branson, S., Anderson, G. A., Bourdev, L.: "Learned Video Compression", Electrical Engineering and Systems Science - Image and Video Processing arXiv:1811.06981v1, 1-11, 2018.

[5] The Moving Picture Experts Group: “MPEG”, URL: https://mpeg.chiariglione.org/ (last request: 2020-09-24).

[6] Zhang, T., Mao, S.: "An overview of emerging video coding standards", GetMobile 22 (4), 13-20, 2018. doi: $10.1145 / 3325867.3325873$

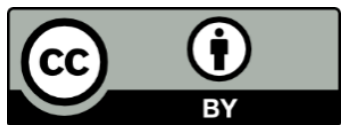

(C) 2020 Authors. Published by the University of Novi Sad, Faculty of Technical Sciences, Department of Graphic Engineering and Design. This article is an open access article distributed under the terms and conditions of the Creative Commons Attribution license 3.0 Serbia (http://creativecommons.org/licenses/by/3.0/rs/). 